\title{
Petit dictionnaire de droit de la santé et de bioéthique
}

\section{Jean Martin}

Dr méd., membre de la rédaction

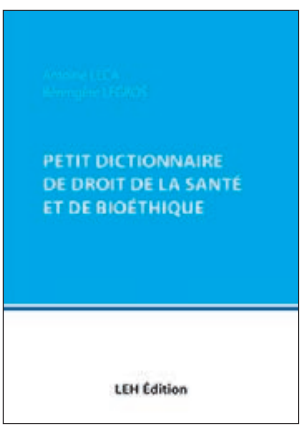

Antoine Leca et Bérengère Legros

Petit dictionnaire de droit de la santé et de bioéthique

Bordeaux: LEH Edition, 2017;

245 pages. $46.50 \mathrm{CHF}$.

ISBN 978-2-84874-689-0

Deux enseignants universitaires de droit de la santé, de Aix-Marseille et Lille, publient cet ouvrage comptant plus de 600 entrées. «Les auteurs décortiquent les pratiques et concepts qui s'épanouissent dans le secteur de la santé humaine», dit la présentation. Ceci sur un large éventail de domaines: pratique et techniques médicales, professions de la santé et des soins, recherche dans différents registres, pharmacie, bioéthique dans ses multiples dimensions actuelles. Pour les aspects légaux est traité essentiellement le cadre français, avec toutefois des indications sur ce qui vaut dans d'autres systèmes. Ainsi on y trouve des informations sur la situation suisse (voir par ex. pp. 163-164 - ailleurs est mentionné l'article de notre Constitution traitant des médecines complémentaires). Les institutions du domaine occupent une place notable. C'est aussi le cas des techniques de soins alternatives. De nombreux médicaments particuliers ont leur rubrique.

Certaines entrées sont très succinctes: toxicodépendance a deux lignes, drogue est traitée dans son sens ancien de produit pharmaceutique. Pas d'entrée sous autonomie ni consentement, pourtant incontournables en déontologie et éthique - mais on trouve registre des refus (de prélèvement d'organes). Deux pages ${ }^{1}$ pour directives anticipées, une pour obstination déraison- nable, mais deux lignes seulement pour clause de conscience. Deux pages pour anonymat.

Pas de rubriques sida, transplantation, suicide ou assistance au suicide. Euthanasie par contre occupe légitimement deux pages et sédation trois (y compris sédation terminale telle qu'introduite par la loi ClaeysLeonetti de février 2016). Trois pages aussi pour soins palliatifs, une pour fin de vie. Nombreuses entrées en rapport avec l'assistance médicale à la procréation. On trouve "disease mongering" ("fabrication" de maladies, sur la base d'intérêts commerciaux), alicament, cannabis thérapeutique ou encore CRISPR-Cas9 - montrant le caractère "up to date» de la rédaction. Le choix de certaines rubriques peut surprendre au premier abord: ainsi restauration tégumentaire (obligation de recoudre décemment la peau après prélèvement d'organes ou autopsie).

Cet ouvrage se parcourt avec intérêt, ses entrées apportent des informations utiles et actuelles, formulant des positions/appréciations équilibrées. Le praticien de la santé publique et de la bioéthique qui rédige la présente analyse a procédé par sondages. Si certains sujets sont traités de manière brève, cela reflète des choix que devaient faire les auteurs - et n'enlève rien au fait que ce «Petit dictionnaire» soit tout à fait utile. Il est aisé à consulter et les professionnels médicaux et des soins, de l'éthique et du droit trouveront pratique de l'avoir à disposition.

Sur un plan général, une question vient à l'esprit: savoir combien de temps des documents papier garderont la place qu'ils ont eue jusqu'ici de références, tout en satisfaisant à des critères d'actualité et de maniabilité - à l'heure où chaque ado a sur son iPhone potentiellement accès à tant de façons d'obtenir aisément des réponses. Reste toutefois la question de la qualité/ sécurité des informations, mieux assurée jusqu'ici par un livre - et par sa maison d'édition. Cela étant, même si à l'avenir des sommes fiables de données pourront de plus en plus souvent être téléchargées à partir de supports numériques, il y a pour l'instant une place et une clientèle pour des ouvrages comme ce dictionnaire - y compris ceux qui apprécient d'avoir en main un «objet de lecture» agréable à consulter. 